\title{
Solid phase antibody assay by means of enzyme conjugated to anti-immunoglobulin
}

\author{
P. LEINIKKI AND SUVI PÄSSILÄ
}

From the Virus Laboratory of the Municipal Bacteriological Laboratory, Aurora Hospital, Helsinki, Finland

SYNOPSIS A solid phase antibody assay by means of alkaline phosphatase conjugated to anti- $-\overline{\frac{\Omega}{O}}$ immunoglobulin is described. Specially designed microcuvettes were sensitized with influenza $A_{i}$ antigen, and antibodies bound to it were assayed by anti-IgG alkaline phosphatase conjugate in $\mathrm{a}_{-}^{\ominus}$ semiautomated photometer equipped with a programmable calculator. The sensitivity was found? to be 200 times higher than HI- or CF-techniques, and the interassay variation was so small that $\vec{\overrightarrow{ }}$ twofold changes in antibody activity could be regarded as significant. Results from vaccinees $\sigma$ indicated that serum samples could be collected at intervals of three to six days only to reach a $\stackrel{\circ}{-}$ serological diagnosis in clinical patients.

Radioimmune assays have found several applications in diagnostic laboratory medicine. They are sensitive, the amount of detectable proteins is measured in nanograms and their versatility allows various experimental arrangements. Their obvious drawbacks are the potential hazards due to ionizing radiation from the reagents and the expensive instrumentation required. Several investigators have developed immunological assays where enzymes instead of radioisotopes are conjugated to antigens or antibodies (Engvall and Perlmann, 1971; Engvall et al, 1971; Engvall and Perlmann, 1972; Avrameas, 1969). These techniques seem to share the versatility and sensitivity of radioimmune assays but lack the abovementioned disadvantages. According to recent studies, they seem suitable for antibody assays in diagnostic microbiology (Carlsson et al, 1972; Holmgren and Svennerholm, 1973; Voller et al, 1974; Voller and Bidwell, 1975). In virology, the quantitative measurement of the reaction and stability of the reagents make the often cumbersome variability of results seen in conventional antibody diassays less likely to occur with this technique.

By using influenza $\mathrm{A}$ as a model, we have developed a solid phase enzyme-linked immunosorbent assay which is suitable for both diagnostic work and largescale epidemiological investigations. Viral antigen is attached to disposable microcuvettes and the amount of fixed antibody is assayed by using alkaline phosphatase coupled to anti-immunoglobulin. The yellow colour resulting from the conversion of the added

Received for publication 28 April 1976 substrate is measured directly from the same cuvettes by a special spectrophotometer.

\section{Material and methods}

ATTACHMENT OF ANTIGEN TO THE CUVETTES The tests were carried out in disposable polystyrene cuvettes developed by Finnpipette-Labsystems, Helsinki, Finland. The tubes were attached onto a nine-hole plate to build a nine-microcuvette block in $\overrightarrow{\hat{O}}$ order to help the handling and the measurement $\exists$ (fig 1).

The tubes were sensitized with influenza $A 2 \stackrel{\text { ? }}{\circ}$ England/74 grown in chicken embryos and purified from allantoic fluid by sucrose band centrifugation:(Orion Diagnostica Laboratories, Mankkaa, Finland). After removal of the sucrose the antigen stock solution was sonicated and diluted into phosphate buffered saline (PBS) to give $25 \mathrm{HA}$ units in $250 \mu 1$. $\mathrm{O}$ This was added to each cuvette and incubated at $33^{\circ} \mathrm{C}$ for 4 hours. They were then washed with PBSO containing $0.05 \%$ Tween 20 (PBS-T) and rinsed with distilled water. Unless used immediately the cuvettes $N$ were stored dry at $+4^{\circ} \mathrm{C}$ until used.

PATIENTS' SERA

Paired serum samples from 17 adult patients and single serum samples from 17 healthy children aged 8-12 months were tested. They had been pretested $\stackrel{\$}{\infty}$ with haemagglutination inhibition (HI), complement ${ }^{-}$ fixation (CF), and single radial haemolysis tech-ō niques (obtained by courtesy of Kari Penttinen, Department of Virology, University of Helsinki). 


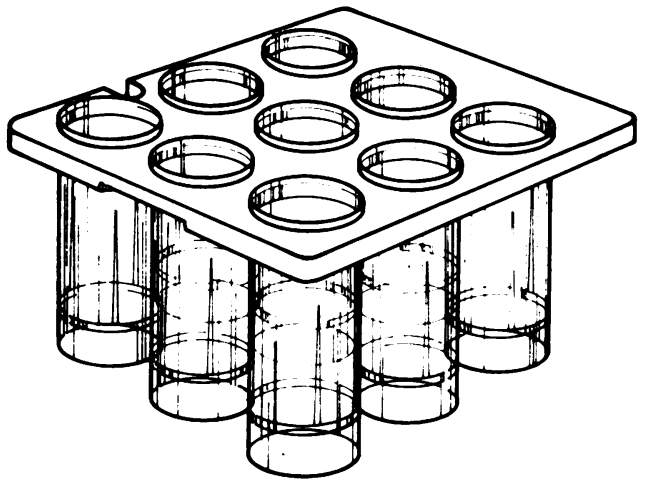

Fig 1 The microcuvette block (Finnpipette ${ }^{R}$ Labsystems) used for antibody assays after sensitization with viral antigen.

One convalescent adult serum which, when pretested, showed antibody activity was chosen as the reference standard serum. In each antibody assay it was serially diluted in half logarithm steps from $10^{-1}$ to $10^{-5}$, and a standard curve was drawn based on the results from this serum. Other sera were assayed at dilutions of 1:50, 1:500, and 1:5000. All sera and the conjugate were diluted into PBS-T and the dilutions were made by using 1-, 3-, and 9-channel disposable tip precision pipettes (Finnpipette ${ }^{\mathrm{R}}$, Helsinki, Finland).

\section{ANTI-IMMUNOGLOBULIN CONJUGATE}

Swine antihuman globulin obtained from Orion Diagnostica was used. It showed high activity against human IgG but no activity against light immunoglobulin chains. It was further purified by being adsorbed to Sepharose 4B covalently linked with human gamma-globulin which was obtained by the sulphate precipitation method from concentrated human gamma-globulin (Finnish Red Cross, Helsinki, Finland). The elution was carried out by lowering the $\mathrm{pH}$ by $0.2 \mathrm{M}$ acetic acid, and the eluted fractions were immediately neutralized with TRIS. After dialysis against PBS the solution was concentrated to $5 \mathrm{mg}$ protein $/ \mathrm{ml}$ and conjugated with alkaline phosphatase (Sigma type VII) with glutardialdehyde, as described by Engvall et al (1971).

Fresh paranitrophenylphosphate (PNPP) solution was used as the substrate. It was prepared by dissolving $150 \mathrm{mg}$ PNPP powder into $25 \mathrm{ml}$ distilled water and was mixed with $25 \mathrm{ml} 0 \cdot 1 \mathrm{M}$ glycine- $\mathrm{MgCl}_{2}$ buffer $\mathrm{pH} 10 \cdot 5$.

\section{ANTIBODY ASSAYS}

Of each serum dilution $200 \mu \mathrm{l}$ was pipetted into cuvettes and incubated for one hour at $+33^{\circ} \mathrm{C}$. After incubation the cuvettes were washed with PBS-T for 10 minutes and rinsed twice with distilled water; $175 \mu \mathrm{l}$ of the conjugate, diluted into PBS-T, was then added and incubated at room temperature overnight. The washing was carried out as previously and $150 \mu \mathrm{l}$ of substrate was added. After $30 \mathrm{~min}$ incubation at $+33^{\circ} \mathrm{C}, 250 \mu \mathrm{l} 0 \cdot 16 \mathrm{M}$ sodium hydroxide was added to stop the reaction.

The optical absorbance was measured using a nine-channel photometer (Finnpipette ${ }^{\mathrm{R}}$ Analyzer System, Finnpipette-Labsystems, Helsinki, Finland) at a wavelength of $405 \mathrm{~nm}$. This photometer measures the absorbance vertically through the bottom of each individual cuvette of the block. The measurement was carried out against sensitized cuvettes treated with conjugate, substrate, and $0.16 \mathrm{M}$ sodium hydroxide as controls. The photometer is equipped with a programmable desk calculator which facilitates the handling of the results.

\section{Results}

CHARACTERIZATION OF THE STANDARD CURVE When the reference serum was assayed in serial dilutions of half-logarithm steps, an S-shaped curve was obtained (fig 2). The nearly linear part extends

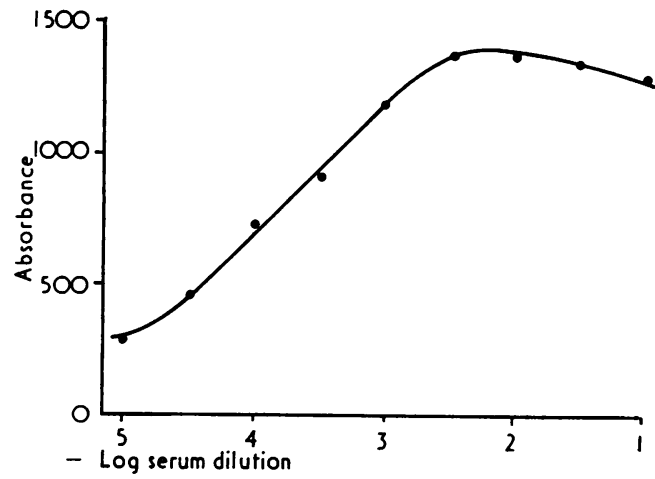

Fig 2 Standard curve obtained from serial dilutions of the reference serum.

from $0 \cdot 3$ to $1 \cdot 2-1 \cdot 4$ absorbance units. This is a major part of the optically effective absorption range. When p-nitrophenol alone is assayed, a linear dose dependence extends from zero to 1.5 absorbance units (data not shown).

The 'levelling out' of the standard curve at the high antibody concentrations evidently reflects a state of saturation where all the available antigen sites have been utilized. The 'levelling out' at low antibody concentrations is probably due to the incapability of the aggregated conjugate to attach onto very sparsely distributed antibodies. 


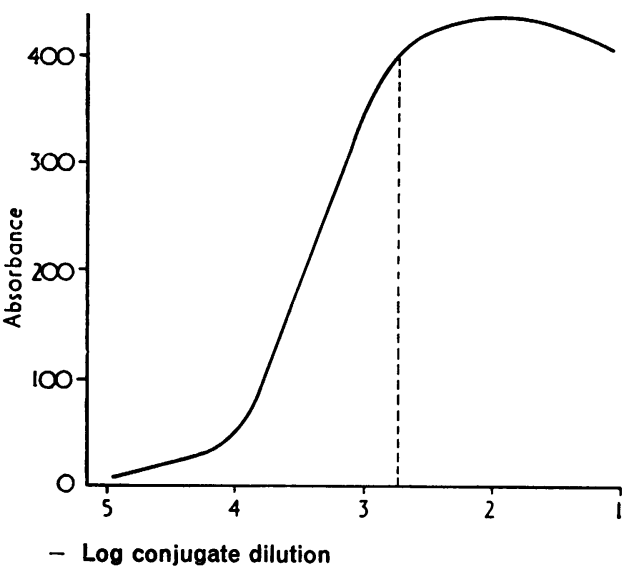

Fig 3 Enzyme activity of serial dilutions of anti-human IgG conjugate in the presence of a known positive human serum diluted to $1 / 5000$.

WORKING DILUTION OF THE CONJUGATE To determine the most suitable working dilution for the conjugate various dilutions of it and a constant dilution of the reference serum were used in the test. High concentrations of the conjugate resulted in a slight prozone effect, but beyond the critical dilution corresponding to the equivalence point a steep decrease was observed (fig 3 ). The working dilution chosen is marked by a dotted line in the figure.

\section{ASSAYS OF INFLUENZA ANTIBODIES FROM PATIENTS' SERA}

To test the reproducibility of the assay 40 parallel dilutions from a serum known to contain influenza antibodies were assayed. Standard deviation was 49.6, which is about $5 \%$ of the effective part of the standard curve. The range for $95 \%$ confidence limits corresponds to about a twofold dilution of the serum as estimated from the standard curve.

The values for the standard curve were seen to fluctuate slightly from test to test. This was probably due to variations in the amounts of antigen absorbed in different lots of sensitized cuvettes and other similar technical variations. Therefore we decided not to interpolate the values obtained for individual sera to anything equivalent to end point titres. Instead we calculated the distance of the observed absorbance values from the standard curve for those dilutions which fell into the linear part of the standard curve (fig 4). The distance was expressed as negative (or positive) Brigg's logarithms (ED-values). For instance, an ED-value of -1.00 indicates that this serum contained one-tenth of the activity of the reference serum. The calculator is easily programmed to give this type of data expression. A majority of acute phase sera from adult patients gave values from

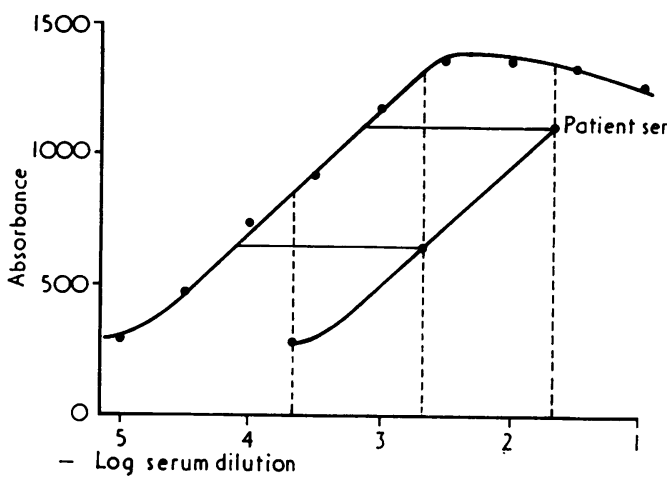

Fig 4 The principle for the estimation of ED-values. $E D$-values express the distance (in log.) of the observed adsorption values of the patient's serum from the standar curve (slim lines). The dotted lines show the dilutions which are routinely used for serum samples to be tested $(1 / 50,1 / 500,1 / 5000)$

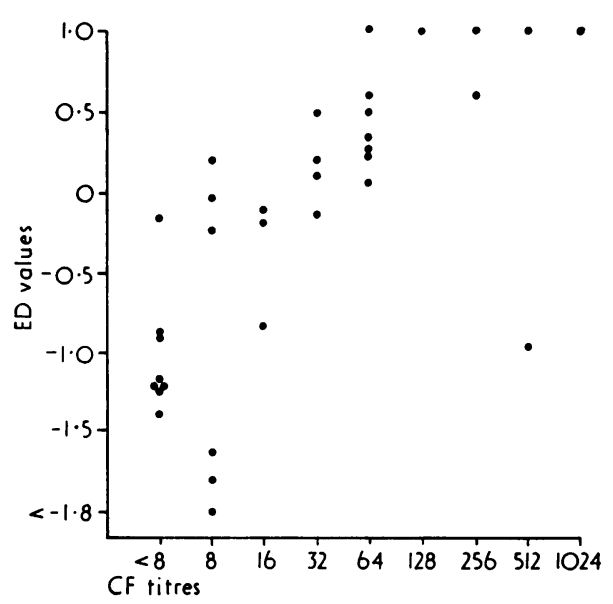

Fig 5 Correlation between complement fixing $(C F)$ and enzyme assay $(E D)$ antibody titres in patients' sera. (ED values $=$ distance from standard curve, see text.$)$

$-1 \cdot 2$ to $-1 \cdot 8$. These values did not seem to indicat an absence of influenza antibodies since sera frome children negative for influenza antibodies with othef techniques gave values from $-1 \cdot 85$ to $-2 \cdot 8$. Most of the convalescent phase sera gave values above 0.18 (figs 5 and 6).

CORRELATION BETWEEN ENZYME ASSAY AND OTHER TESTS

The correlation between ED-values and CF-titres? seemed to be good (fig 5). However, a number of sera negative with CF when tested at a dilution of 1:8 showed definite activity in the enzyme assay? reflecting the greater sensitivity of the enzyme assa 8 


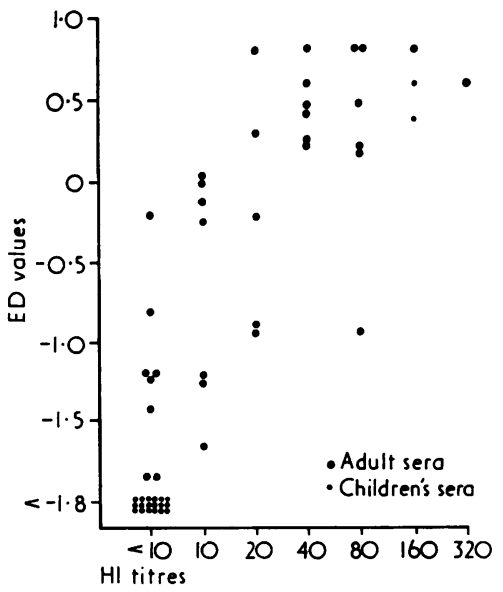

Fig 6 Correlation between haemagglutination inhibition $(H I)$ and enzyme assay $(E D)$ antibody titres in sera from adult patients and healthy children.

compared to CF. In two serum pairs no increase in ED-values was noted in spite of a significant increase in CF- and HI-titres. On the other hand, no significant increase had been found with single radial haemolysis either. These discrepancies may reflect differences in the specificities of the tests. The correlation between ED-values and HI-titres seemed very similar (fig 6). Two of the children's sera tested gave high ED-values. They were also the only HIpositive samples among them.

\section{STUDIES ON VACCINEES}

In order to see whether the interval between serum samples in clinical patients could be shortened nine vaccinees were studied. They were vaccinated with killed influenza A2 vaccine (Flupar ${ }^{\mathrm{R}}$, Orion Diagnostica, Finland) and serum samples were taken at days $0,3,6,9$, and 12 . In two vaccinees the enzyme assay showed a significant increase between samples taken at days 0 and 3 . They had been vaccinated with the same vaccine one year earlier. In three cases, the increase could be demonstrated in samples taken at days 3 and 6; none of these had been vaccinated before. Vaccinees with high initial antibody activity did not show any further increase in antibody activity.

\section{Discussion}

Several earlier reports indicate that antibody assays based on enzyme markers reach a sensitivity equal to radioimmunological assays (Avrameas, 1969; Engvall and Perlmann, 1972). In our model, the endpoint titre of the reference serum can be estimated from the point where the standard curve crossed the linear, which is two times standard deviation above the background activity. This gives a titre of $1: 70000$ to the serum while its HI-titre was 320 and CF-titre 256. A rough estimate would indicate that our assay is about 200 times more sensitive than the conventional techniques.

The measurement of the enzyme activity directly through the bottoms of the sensitized cuvettes did not seem to increase the background absorbance significantly. This could be ascertained by using non-sensitized cuvettes containing sodium hydroxide as the blank. On the other hand, the possibility of carrying out the complete assay in the same cuvettes simplified the procedure and increased its accuracy.

A virological diagnosis based on an increase in antibody activity during the early phases of the illness can be performed if sufficient amounts of circulating antibodies are produced. The sensitivity and accuracy of this assay would enable a shortening in the interval between serum samples from clinical patients. Studies on vaccinees showed that a significant increase can be demonstrated within three to six days from the onset of the antigenic stimulus. We have also had an opportunity to analyse serum samples from a few clinical influenza patients collected at short intervals. The results indicate that a significant increase in antibody activity can be demonstrated within five days from the onset of the disease. In most cases, especially in adults, influenza A infection is a reinfection, and clinical materials from other infections must be analysed to see whether this type of rapid diagnosis can be achieved in them also.

Another prerequisite for this type of rapid diagnosis is that fluctuation in antibody activity does not occur in an individual suffering from clinical illnesses due to unrelated microorganisms. For example, we have analysed paired serum samples for influenza antibody from a number of patients who had various types of upper and lower respiratory illnesses not due to influenza $A$. The change in ED-values in samples taken 10 days apart was less than $0 \cdot 10$. This seems to indicate that although the assay is sensitive, a background fluctuation will not hamper the serological diagnosis.

Since the assay can be performed within 24 hours it can be of considerable help in diagnostic virus laboratories. Furthermore, sensitized cuvettes retain their activity for months and the conjugate even longer. This technique is also applicable to largescale epidemiological works. Multichannel precision pipettes with disposable tips and automation of the optical measurement makes possible the handling of large numbers of sera in each series. Highly purified antigens can be used for special purposes 
while rather crude, commercially available antigens seem to be suitable for routine diagnostic work (Voller and Bidwell, 1975). In order to get comparable results in different laboratories a reference serum would be needed with a known amount of specific antibodies. As soon as this was available, the antibody activity could be expressed in nanograms of antibody for the antigen used.

By using enzyme conjugated to anti-IgM this technique can be used to demonstrate ear!y phase antibodies in patients' sera. In preliminary experiments this assay seems to be very suitable but the problems from competition by IgG antibodies and the anti-IgG activity of some IgM antibodies must be solved before it can be applied to routine clinical use.

This work was supported by the Sigrid Jusélius Foundation. We wish to thank Eva Engvall, MD, for valuable advice, Professor Kari Penttinen, MD, for the pretested sera, Jukka Suni, MD, who carried out the vaccination, Osmo Suovaniemi, MD, from Finnpipette-Labsystems Inc for putting the cuvettes and the spectrophotometer at our disposal, and Orion Diagnostica Inc for giving us the influenzaantigen.

\section{References}

Avrameas, S. (1969). Coupling of enzymes to proteins with glutaraldehyde: use of the conjugates for the detection of antigens and antibodies. Immunochemistry, 6, 43-52.

Carlsson, H., Lindberg, A., and Hammarström, S. (1972)등 Titration of antibodies to salmonella $O$ antigens byes enzyme-linked immunosorbent assay. Infect. Immun., 6 . 703-708.

Engvall, E., Jonsson, K., and Perlmann, P. (1971). Enzymeџ linked immunosorbent assay (ELISA). II. Quantitative assay of protein antigen immunoglobulin $G$ by means o $\vec{B}$ enzyme-labelled antigen and antibody-coated tubes:Biochem. biophys. acta, 251, 427-434.

Engvall, E. and Perlmann, P. (1971). Enzyme-linked immuno. sorbent assay (ELISA). Quantitative assay of immunoglobulin G. Immunochemistry, 8, 871-874.

Engvall, E. and Perlmann, P. (1972). Enzyme-linked immunoê sorbent assay, ELISA. III. Quantitation of specific antibodies by enzyme labeled anti-immunoglobulin in antigen $N$ coated tubes. J. Immunol., 109, 129-135.

Holmgren, J. and Svennerholm, A. M. (1973). Enzyme $\vec{\sigma}$ linked immunosorbent assay for cholera serology. Infecto Immun., 7, 759-763.

Voller, A. and Bidwell, D. E. (1975). A simple method for detecting antibodies to rubella. Brit. J. exp. Path., 56 338-340.

Voller, A., Bidwell, D., Huldt, G., and Engvall, E. (1974). A microplate method of enzyme-linked immunosorben assay and its application to malaria. Bull. Wld Hlth Org. $\frac{\mathbb{\Phi}}{-}$ 51, 209-211. 